\title{
The spin and orbit of the newly discovered pulsar IGR J17480-2446
}

\author{
A. Papitto ${ }^{1}$, A. D’ Aì ${ }^{2}$, S. Motta ${ }^{3}$, A. Riggio ${ }^{1,4}$, L. Burderi ${ }^{1}$, T. Di Salvo ${ }^{2}$, T. Belloni ${ }^{3}$, and R. Iaria ${ }^{2}$ \\ 1 Dipartimento di Fisica, Università degli Studi di Cagliari, SP Monserrato-Sestu, KM 0.7, 09042 Monserrato, Italy \\ e-mail: apapitto@oa-cagliari.inaf.ig \\ 2 INAF - Osservatorio Astronomico di Cagliari, Poggio dei Pini, Strada 54, 09012 Capoterra (CA), Italy \\ 3 Dipartimento di Scienze Fisiche ed Astronomiche, Università di Palermo, via Archirafi 36, 90123 Palermo, Italy \\ ${ }^{4}$ INAF - Osservatorio Astronomico di Brera, via E. Bianchi 46, 23807 Merate, Italy \\ Received 21 October 2010 / Accepted 28 November 2010
}

\section{ABSTRACT}

\begin{abstract}
We present an analysis of the spin and orbital properties of the newly discovered accreting pulsar IGR J17480-2446, located in the globular cluster Terzan 5. Considering the pulses detected by the Rossi X-ray Timing Explorer at a period of 90.539645(2) ms, we derive a solution for the $21.27454(8) \mathrm{hr}$ binary system. The binary mass function is estimated to be $0.021275(5) M_{\odot}$, indicating a companion star with a mass larger than $0.4 M_{\odot}$. The X-ray pulsar spins up while accreting at a rate of between 1.2 and $1.7 \times$ $10^{-12} \mathrm{~Hz} \mathrm{~s}^{-1}$, in agreement with the accretion of disc matter angular momentum given the observed luminosity.

We also report the detection of pulsations at the spin period of the source during a Swift observation performed $\sim 2 \mathrm{~d}$ before the beginning of the RXTE coverage. Assuming that the inner disc radius lies in between the neutron star radius and the corotation radius while the source shows pulsations, we estimate the magnetic field of the neutron star to be within $\sim 2 \times 10^{8} \mathrm{G}$ and $\sim 2.4 \times 10^{10} \mathrm{G}$. From this estimate, the value of the spin period and of the observed spin-up rate, we associate this source with the still poorly sampled population of slow, mildly recycled, accreting pulsars.
\end{abstract}

Key words. stars: neutron - stars: rotation - X-rays: binaries - pulsars: individual: IGR J17480-2446

\section{Introduction}

The dense environment of a globular cluster and the resulting frequent stellar encounters (Meylan \& Heggie 1997) make the production of binary systems hosting a compact object very efficient. Terzan 5 is probably one of the densest and metal-richest cluster in our Galaxy (Cohn et al. 2002; Ortolani et al. 2007), as clearly indicated by the large number of rotation-powered millisecond pulsars discovered there $(z 30$, Ransom et al. 2005; Hessels et al. 2006). The cluster also contains at least 28 discrete $\mathrm{X}$-ray sources, a substantial number of which can be identified as quiescent low-mass X-ray binaries (LMXB, Heinke et al. 2006). According to the recycling scenario (see, e.g. Bhattacharya \& van den Heuvel 1991), the population of rotation-powered millisecond pulsars and low-mass X-ray binaries (LMXB) share an evolutionary link, because the former are thought to be spun up by the accretion of mass and angular momentum in an LMXB. Accreting pulsars in LMXB are usually found with periods clustering in two distinct groups. So far, 13 sources have been discovered with spin periods lower than $10 \mathrm{~ms}$ and were therefore termed accretion-powered millisecond pulsars (see, e.g., Wijnands \& van der Klis 1998). However, a smaller number of sources are found with longer periods and correspondingly higher estimates of the neutron star (NS) magnetic field.

So far, the only bright transient LMXB known in the cluster Terzan 5 was the burster EXO 1745-248 (Makishima et al. 1981). The first detection of a new outburst of a source in this cluster was made on 2010 October 10.365 with INTEGRAL (Bordas et al. 2010) and was tentatively attributed to EXO 1745-248. Follow-up Swift observations refined the source position, and a comparison with the position of sources previously known thanks to Chandra observations of the cluster suggested instead a different association (Heinke et al. 2010;
Kennea et al. 2010; Pooley et al. 2010). The X-ray transient is then considered a newly discovered source and named as IGR $\mathrm{J} 17480-2446$ (J17480 in the following). A coherent signal at a period of $90.6 \mathrm{~ms}$ was detected thanks to observations performed with the Rossi X-ray Timing Explorer (RXTE in the following, Strohmayer \& Markwardt 2010). A signal at the same period is present also during the several bursts that the source shows (Altamirano et al. 2010b), while burst oscillations have never been observed from NS rotating at periods exceeding a few ms. A sudden decrease of the flux was tentatively attributed to an eclipse of the source by the companion (Strohmayer \& Markwardt 2010). However, eclipses were not observed during subsequent observations, and the earlier flux decrease was identified with a lunar occultation (Strohmayer et al. 2010, S10 in the following).

Below we present the first analysis of the properties of the coherent signal emitted by this source, using $R X T E$ and Swift observations performed between 2010 October 10 and November 6, and give a refined orbital and timing solution of the pulsar with respect to those first proposed (Papitto et al. 2010, P10 in the following; S10).

\section{Observations and data analysis}

After the source discovery on 2010 October 10.365 (Bordas et al. 2010), RXTE started monitoring the source on MJD 55482.010 (October 13.010; all the epochs reported in this paper are given with regard to the Barycentric Dynamical Time, TDB, system). We present an analysis of the observations performed until MJD 55506.359 (ObsId 95437-01-12-01), for a total exposure of $206 \mathrm{ks}$. In this time interval, a large number $(>300)$ of X-ray bursts are observed with a recurrence time decreasing from $\gtrsim 26$ to $\sim 3 \mathrm{~min}$ as the X-ray flux increases. The analysis of the bursts 


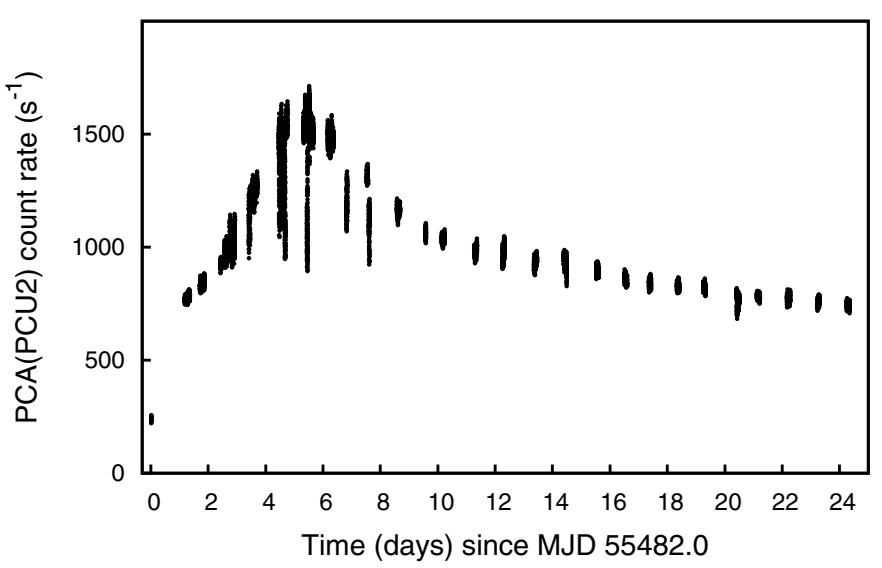

Fig. 1. J17480 in the time interval considered here as observed by the PCU2 of the PCA aboard RXTE.

shown by the source, as well as of its aperiodic timing properties, will appear in a companion paper. The $2.5-25 \mathrm{keV}$ light curve recorded by PCU2 of the Proportional Counter Array (PCA) on-board RXTE, with the burst intervals removed and background subtracted, is plotted in Fig. 1. The count rate increases during the first days of the outburst, reaching a peak value of $\sim 1700 \mathrm{~s}^{-1}$ at MJD 55 487.5, and then decreases to a value of $\sim 800 \mathrm{~s}^{-1}$ with an exponential decay time scale of $\sim 5 \mathrm{~d}$. Quite complex dipping-like structures also appear especially between MJD 55485 and 55490 as the flux shows sudden variations up to $75 \%$ on timescales of $\sim 10 \mathrm{~min}$.

The combined X-ray spectrum observed by the top layer of the PCU2 of the PCA (2.5-25 keV), and by Cluster A of the High Energy X-ray Timing Experiment (HEXTE, 22-50 keV), can be well modelled by a sum of a blackbody and a Comptonized component, which we model with compps (Poutanen \& Svensson 1996). Throughout the observations considered here, the spectrum softens significantly as the source evolves towards higher luminosities. The unabsorbed total flux, extrapolated in the $0.1-100 \mathrm{keV}$ band, rises from $0.47(3) \times 10^{-8}$ to a maximum level of $1.89(4) \times 10^{-8} \mathrm{erg} \mathrm{cm}^{-2} \mathrm{~s}^{-1}$, observed during the observation of MJD 55 487.5. All the uncertainties on the fluxes given here are quoted at a $90 \%$ confidence level.

To analyse the spin and orbital properties of the source we consider data taken by the PCA in Event (122 $\mu$ s temporal resolution) and Good Xenon ( $1 \mu$ s temporal resolution) packing modes. We discard $10 \mathrm{~s}$ prior, and $100 \mathrm{~s}$ after the onset of each type-I X-ray burst. The time series were also preliminarly barycentred with respect to the solar system barycentre using the available RXTE orbit files and assuming the best Chandra estimate of the source position, RA $=17^{\mathrm{h}} 48^{\mathrm{m}} 4.831^{\mathrm{s}}$, Dec $=-24^{\circ} 46^{\prime} 48.87^{\prime \prime}$, with an error circle of $0.06^{\prime \prime}$ ( $1 \sigma$ confidence level, Heinke et al. 2006; Pooley et al. 2010). A coherent signal at a frequency of $11.045(1) \mathrm{Hz}$ [equivalent to a period of $90.539(8) \mathrm{ms}$ ] is clearly detected in the power spectrum at a Leahy-normalised power of $\simeq 1.5 \times 10^{4}$. A first orbital solution is obtained modelling the observed modulation caused by the orbital motion,

$P(t)=P_{0}\left\{1+\frac{2 \pi x}{P_{\text {orb }}}[\cos m+e \cos (2 m-\omega)]\right\}$.

Here $P_{0}$ is the barycentric spin period of the source, $x=a \sin i / c$ the semi-major axis of the NS orbit, $P_{\text {orb }}$ the orbital period, $m=2 \pi(t-T *) / P_{\text {orb }}$ the mean anomaly, $T^{*}$ the epoch at which $m=0, e$ the eccentricity and $\omega$ the longitude of the periastron measured from the ascending node. The periods $P(t)$ are
Table 1. Spin and orbital parameters of IGR J17480-2446.

\begin{tabular}{lccc}
\hline \hline & Periods & 1st harm. ph. & 2nd harm. ph. \\
\hline$v_{0}-11.044885(\mu \mathrm{Hz})$ & $<5$ & $+0.64(1)$ & $+0.17(1)$ \\
$\langle\dot{v}\rangle\left(10^{-12} \mathrm{~Hz} \mathrm{~s}^{-1}\right)$ & $<16$ & $1.22(1)$ & $1.68(1)$ \\
\hline$a \sin i / \mathrm{c}(\mathrm{lt}-\mathrm{s})$ & $2.498(5)$ & $2.4967(3)$ & $2.4973(2)$ \\
$P_{\text {orb }}(\mathrm{hr})$ & $21.2744(8)$ & $21.2745(1)$ & $21.27454(8)$ \\
$T *-55481.0(\mathrm{MJD})$ & $0.7805(4)$ & $0.78033(6)$ & $0.78048(4)$ \\
$e$ & $<0.02$ & $<7 \times 10^{-4}$ & $<6 \times 10^{-4}$ \\
$f\left(M_{1}, M_{2}, i\right)\left(M_{\odot}\right)$ & $0.0213(2)$ & $0.0212587(8)$ & $0.021275(5)$ \\
\hline$\chi^{2} /$ d.o.f. & $156 / 121$ & $7879 / 662$ & $3287 / 566$ \\
\hline
\end{tabular}

Notes. Numbers in parentheses are $1 \sigma$ errors on the last significant digit. Upper limits are evaluated at $3 \sigma$ confidence level. The uncertainties of the timing parameters have been scaled by a factor $\sqrt{\chi_{\mathrm{r}}^{2}}$ to take into account a reduced $\chi^{2}$ of the best-fitting model larger than 1 . The orbital solution obtained modelling the Doppler shifts on the pulse period is given in the leftmost column, while the best timing solution evaluated from the phase evolution of the first and second harmonic are given in the central and in the rightmost column, respectively. The reference epoch for these timing solutions is MJD 55 483.186.

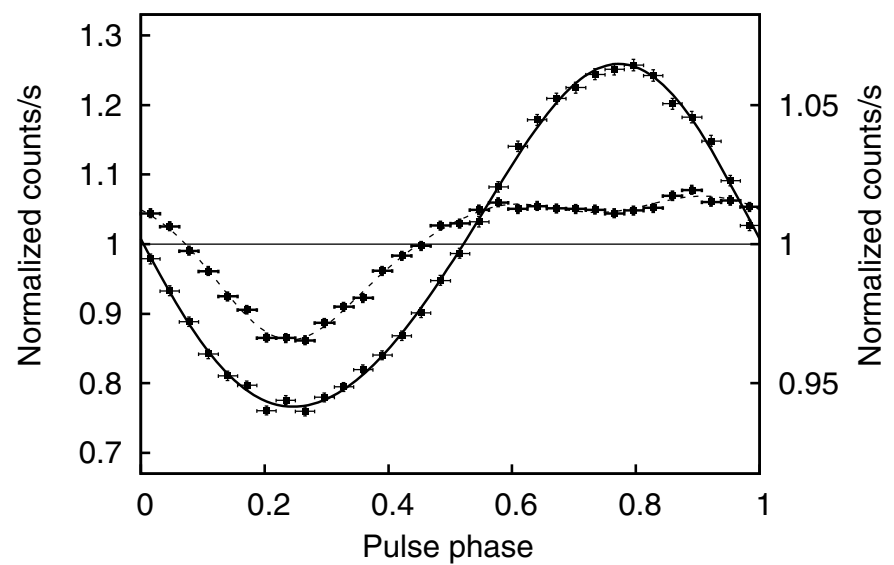

Fig. 2. Pulse profiles and best-fitting harmonic decomposition, obtained by folding observations performed by RXTE on MJD 55482 (left scale, solid line) and MJD 55483 (right scale, dashed lines) around $P_{\mathrm{F}}=$ $90.539645 \mathrm{~ms}$. Both profiles are evaluated after background subtraction and are normalised to the current average flux.

estimated by performing an epoch-folding search on $1.5 \mathrm{ks}$ long data segments (for a total of 127) around the periodicity indicated by the power spectrum. The resulting variance profiles are fitted following Leahy (1987) and the uncertainties affecting the period estimates are evaluated accordingly. The best-fitting orbital solution we obtain with this technique is shown in the leftmost column of Table 1 .

The time series were then corrected for the orbital motion with these parameters and folded around the best estimate of the spin period over $300 \mathrm{~s}$ data segments (for a total of 717). The pulse profiles could generally be modelled using up to three harmonics. The pulsed fraction is observed to greatly vary in between the first (MJD 55482.010 to 55482.043) and the other $R X T E$ observations. During the former the pulse fractional amplitude of the first harmonic is very high $\left[A_{1} \simeq 0.252(2)\right]$, while the second harmonic is detected at a much lower amplitude $\left[A_{2}=0.016(2)\right]$. In subsequent observations the amplitude of the first harmonic drastically decreases to values between 0.02 and 0.04 , whereas the second harmonic amplitude remains stable and a third harmonic is sometimes requested by the profile modelling. To show this, we plot in Fig. 2 the pulse profiles calculated over the observations performed during MJD 55482 (solid line) and MJD 55483 (dashed line), with the latter profile 
shown at a magnified scale. The pulsed fraction decrease is evident, as are the variation of the shape of the peak at rotational phase $\sim 0.75$.

To increase the accuracy of our timing solution we model the temporal evolution of the phases evaluated on the first and second harmonic of the pulse profiles with the relation:

$\phi(t)=\phi_{0}+\left(v_{0}-v_{\mathrm{F}}\right)\left(t-T_{\mathrm{ref}}\right)+\frac{1}{2}\langle\dot{v}\rangle\left(t-T_{\mathrm{ref}}\right)^{2}+R_{\mathrm{orb}}(t)$.

Here $T_{\text {ref }}$ is the reference epoch for the timing solution, $v_{0}$ is the pulsar frequency at the reference epoch, $\nu_{\mathrm{F}}=1 / P_{\mathrm{F}}$ is the folding frequency and $\langle\dot{v}\rangle$ is the average spin frequency derivative. The term $R_{\mathrm{orb}}(t)$ describes the phase residuals induced by a difference between the actual orbital parameters, namely $x, P_{\text {orb }}, T^{*}$, $e \sin \omega$ and $e \cos \omega$, and those used to correct the time series (see e.g. Deeter et al. 1981). Once a new set of orbital parameters is found, it is used to correct the time series, and the resulting phases are modelled again with Eq. (2). This procedure is iterated until the phase residuals are normally distributed around zero.

Although no residual modulation at the orbital period is observed, the phases of the first harmonic are strongly affected by timing noise. The reduced $\chi^{2}$ we obtain modelling their evolution with Eq. (2) is extremely large ( $\simeq 11.9$ over 662 d.o.f.). Such a behaviour is most probably caused by to pulse shape changes like the one shown in Fig. 2. The second harmonic phases appear to be less affected by timing noise, resulting in a reduced $\chi_{\mathrm{r}}^{2}=5.8$ (566 d.o.f.). We argue that the second harmonic phases are better fitted with respect to the first harmonic because of the greater stability of this component (as already observed in some accreting millisecond pulsars, see, e.g., Burderi et al. 2006; Riggio et al. 2008). The best-fitting parameters calculated over the phase evolution of the first and second harmonic are quoted in the central and rightmost column of Table 1. In Fig. 3 we show the phases of both harmonics, when the observations corrected for the orbital motion of the source are folded around $P_{\mathrm{F}}=90.539645 \mathrm{~ms}$. The phase evolution is clearly driven by at least a quadratic component. A consequence of timing noise is that the spin frequency and its derivative, estimated over the two harmonic components, are significantly different. We quote conservatively a value of $v_{0}=11.0448854(2) \mathrm{Hz}$ that overlaps both frequency estimates, and use a spin frequency derivative between $1.2-1.7 \times 10^{-12} \mathrm{~Hz} \mathrm{~s}^{-1}$ in the discussion below. However, it is worthwhile to note that the orbital parameters are entirely consistent between the two harmonic solutions, which supports the reliability of these estimates. The solution we obtained is entirely compatible with, but more precise than, those proposed by $\mathrm{P} 10^{1}$ and $\mathrm{S} 10$. Given the accuracy of the source position considered here $\left(0.06^{\prime \prime}\right)$, the systematic uncertainties introduced by the position indetermination on the measured values of spin frequency and of its derivative (e.g. Burderi et al. 2007) are $\sigma_{\text {pos, } v} \lesssim 3 \times 10^{-10} \mathrm{~Hz}$ and $\sigma_{\text {pos, },} \lesssim 6 \times 10^{-17} \mathrm{~Hz} \mathrm{~s}^{-1}$, respectively. Finally, as the cluster moves towards the solar system at a velocity of $85 \pm 10 \mathrm{~km} \mathrm{~s}^{-1}$ (Ferraro et al. 2009), the measured value of the spin frequency is affected by a systematic offset of $\sim+3 \times 10^{-3} \mathrm{~Hz}$, though this is unimportant when making conclusions about the source properties.

In order to extend the range of fluxes at which the source was observed and pulsations were detected, we also analysed three Swift observations (Obs. 00031841002,00031841003 and

\footnotetext{
1 There is an offset between the values of frequency and epoch of mean longitude quoted by P10 and those presented here, as theirs were not referred to the TDB reference system.
}

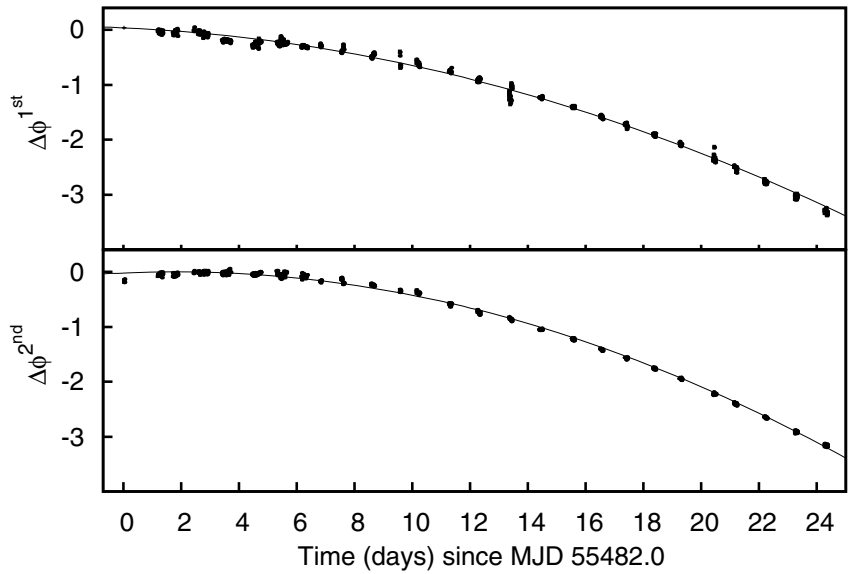

Fig. 3. Evolution of the first (top) and second (bottom) harmonic phases obtained by folding the orbitally corrected time series around $P_{\mathrm{F}}=$ $90.539645 \mathrm{~ms}$ (see text). The solid lines show the best-fit models from Eq. (2).

00031841004) in which the XRT observed in Windowed Timing (WT) mode, with a temporal resolution of $1.7 \mathrm{~ms}$. The Swift XRT started monitoring the source on MJD 55479.737, more than two days before RXTE, with the first observation in WT mode starting at MJD 55479.802 for $2 \mathrm{ks}$. After applying barycentric corrections for the satellite orbit, then correcting for the source orbital motion and selecting photons from a 50 pixel wide box around the source position, a pulsation is clearly detected at a period of $P_{\mathrm{S}}=90.5395(2) \mathrm{ms}$ by means of an epoch-folding search. The XRT signal is particularly strong and consistent with that seen by $R X T E$ during its first observation, with a pulse profile modelled by a sinusoid of amplitude 0.23(2). Pulsations were also searched for in the subsequent $\sim 1 \mathrm{ks}$ long XRT WT observations starting on MJD 55484.767 and 55 485.357. Only a weak signal was detected in the latter at a fractional amplitude of $0.018(4)$, still compatible with that seen by RXTE at those later times.

\section{Discussion and conclusions}

We reported on the spin and orbital properties of the newly discovered accreting pulsar, IGR J17480-2446. Its $90.6 \mathrm{~ms}$ period makes it the first confirmed accreting pulsar in the range 10-100 ms. Pulsations were detected in all observations performed by RXTE, as well as in two out of the three Swift observations performed in WT mode presented here. The pulsed fraction is observed to drastically change on a timescale of $\lesssim 1 \mathrm{~d}$, after the RXTE observation performed on MJD 55482 . While previously both Swift and RXTE observations revealed a strong signal dominated by a first harmonic component of fractional amplitude as large as 0.25 , later observations at higher fluxes performed by both satellites never detected an amplitude $\gtrsim 0.03$. Simultaneously the pulse shape changes and becomes more complex. This behaviour is suggestive of a change of the geometrical properties of the flow in the accretion columns above the NS hot spots.

Because pulsations are detected throughout the observations shown here, an estimate of the NS magnetic field strength can be made. For accretion to proceed and pulsations to appear in the X-ray light curve, the inner disc radius, $R_{\text {in }}$, has to lie in between the NS radius, $R_{\mathrm{NS}}$, and the corotation radius, $R_{\mathrm{C}}$, defined as the distance from the NS at which the velocity of the magnetosphere equals the Keplerian velocity of the matter in the disc. 
For a larger accretion radius, accretion would be inhibited or severely reduced by the onset of a centrifugal barrier. For a pulsar spinning at $90.6 \mathrm{~ms}, R_{\mathrm{C}}=\left(G M P^{2} / 4 \pi^{2}\right)^{1 / 3}=338 m_{1.4}^{1 / 3} \mathrm{~km}$, where $m_{1.4}$ is the NS mass in units of $1.4 M_{\odot}$. Defining the inner disc radius in terms of the pressure equilibrium between the disc and the magnetosphere, one obtains $R_{\text {in }} \simeq 160 \mathrm{~m}_{1.4}^{1 / 7}$ $R_{6}^{-2 / 7} \quad L_{37}^{-2 / 7} \mu_{28}^{4 / 7} \mathrm{~km}$ (Burderi et al. 2001), where $R_{6}$ is the NS radius in units of $10 \mathrm{~km}, L_{37}$ the accretion luminosity in units of $10^{37} \mathrm{erg} \mathrm{s}^{-1}$, and $\mu_{28}$ the magnetic dipole moment of the NS in units of $10^{28} \mathrm{G} \mathrm{cm}^{3}$. Extrapolating the fluxes observed by RXTE to the $0.1-100 \mathrm{keV}$ band, and assuming a distance of $d=5.5 \pm 0.9 \mathrm{kpc}$ to Terzan 5 (Ortolani et al. 2007), we estimate a maximum and minimum bolometric luminosities of $1.7(1) \times 10^{37} d_{5.5}^{2} \mathrm{erg} \mathrm{s}^{-1}$ and $6.8(1) \times 10^{37} d_{5.5}^{2} \mathrm{erg} \mathrm{s}^{-1}$, during the time covered by the observations considered here. Assuming that the X-ray luminosity is a good tracer of the accretion power and imposing $R_{\mathrm{NS}}<R_{\text {in }} \lesssim R_{\mathrm{C}}$, we obtain

$0.02 m_{1.4}^{-1 / 4} R_{6}^{9 / 4} d_{5.5} \lesssim \mu_{28} \lesssim 4.8 m_{1.4}^{1 / 3} R_{6}^{1 / 2} d_{5.5}$.

The upper limit on the magnetic dipole can be reduced considering that pulsations are detected also in a Swift observation taking place $\sim 2 \mathrm{~d}$ earlier than the first $R X T E$ observation. Bozzo et al. (2010) estimated the source flux in that observation as $4.5(2) \times 10^{-10} \mathrm{erg} \mathrm{cm}^{-2} \mathrm{~s}^{-1}(1-10 \mathrm{keV})$. This value is a factor $\sim 4$ lower than the value obtained extrapolating the spectrum of the first RXTE observation in the same energy band. Assuming that this ratio holds also for the bolometric luminosity of the source, we get to an upper limit on the magnetic dipole moment of $\simeq 2.4 \times 10^{28} \mathrm{G} \mathrm{cm}^{-3}$. The limits thus derived translate to a magnetic surface flux density between $\sim 2 \times 10^{8}$ and $\sim 2.4 \times 10^{10} \mathrm{G}$. The upper bound of this interval can be overestimated because the exact flux at which the pulsations appeared is unknown at present. Monitoring the presence of coherent pulsations as a function of the flux when the source fades will probably allow us to derive a tighter constraint. Altamirano et al. (2010a) have also reported the presence of a $\mathrm{kHz}$ QPO at $\sim 815 \mathrm{~Hz}(10-50 \mathrm{keV})$ during the RXTE observations performed on MJD 55487 . Under the hypothesis that this feature originates in the innermost part of the accretion disc, it indicates an inner disc radius $R_{\text {in }} \lesssim 20 m_{1.4}^{1 / 3} \mathrm{~km}$. As the luminosity we estimated during that day is $6.8(1) \times 10^{37} d_{5.5}^{2} \mathrm{erg} \mathrm{s}^{-1}$, this would imply a magnetic field $\lesssim 7 \times 10^{8} d_{5.5} \mathrm{G}$ if the disc is truncated at the magnetospheric radius.

Despite the presence of timing noise, the analysis of the phase evolution over the $\sim 24 \mathrm{~d}$ time interval presented here clearly indicates the need for a quadratic component to model these phases. Interpreting this component as a tracer of the NS spin evolution, we thus conclude that the source spins up while accreting. Values of the spin-up rate between 1.2 and $1.7 \times 10^{-12} \mathrm{~Hz} \mathrm{~s}^{-1}$ are found, depending on the harmonic considered. This discrepancy is probably due to the effect of timing noise. These values are compatible with those expected for a NS accreting the Keplerian disc matter angular momentum given the observed luminosity, $\dot{v} \simeq 1.5 \times 10^{-12}\left(L_{37} / 5\right)\left(R_{\text {in }} / 70 \mathrm{~km}\right)^{1 / 2} I_{45}^{-1}$ $R_{6} m_{1.4} \mathrm{~Hz} \mathrm{~s}^{-1}$. Here $I_{45}$ is the NS moment of inertia in units of $10^{45} \mathrm{~g} \mathrm{~cm}^{2}$. The observed spin period and the magnetic field we estimated place this source between the population of "classical" $\left(B \gtrsim 10^{11} \mathrm{G}, P \gtrsim 0.1 \mathrm{~s}\right)$ and millisecond $\left(B \simeq 10^{8}-10^{9} \mathrm{G}\right.$, $P \simeq 1.5-10 \mathrm{~ms})$ rotation-powered pulsars. The observation of a significant spin-up at rates compatible with those predicted by the recycling scenario further supports the identification of this source as a slow, mildly recycled pulsar. We note that the only other two accreting pulsars with similar, though significantly different parameters, are GRO J1744-28 $\left(P_{\mathrm{S}}=467 \mathrm{~ms}\right.$, $B \simeq 2.4 \times 10^{11} \mathrm{G}$, Cui 1997$)$, and $2 \mathrm{~A} 1822-371\left(P_{\mathrm{S}}=590 \mathrm{~ms}\right.$, $B \lesssim 10^{11} \mathrm{G}$, Jonker \& van der Klis 2001).

The orbital parameters we measured for the NS in J17480 allow us to derive constraints on the nature of its companion star. With a mass function of $f\left(M_{2} ; M_{1}, i\right) \simeq 0.02 M_{\odot}$, a minimum mass for the companion can be estimated to be as low as $0.41 M_{\odot}$ for an inclination of $90^{\circ}$, and an NS mass of $1.4 M_{\odot}$. Since the source shows no eclipses, the inclination is most probably $\lesssim 80^{\circ}$, and the lower limit increases to $m_{2}=0.16+0.26 m_{1.4}$, where $m_{2}$ is the mass of the companion star in solar units. An upper limit can be obtained if the companion star is assumed not to overfill its Roche lobe. Using the relation given by Eggleton (1983) and the third Kepler law to relate the Roche Lobe radius to the orbital period and to the companion mass, $R_{L 2} \simeq$ $0.55\left(G M_{\odot}\right)^{1 / 3}\left(P_{\text {orb }} / 2 \pi\right)^{2 / 3} m_{1.4} q^{2 / 3}(1+q)^{1 / 3} /\left[0.6 q^{2 / 3}+\log (1+\right.$ $\left.q^{1 / 3}\right)$, where $q=M_{2} / M_{1}$, and assuming the companion star follows a main sequence mass-radius relation, $R_{2} / R_{\odot} \approx\left(M_{2} / M_{\odot}\right)$, yields a maximum mass of $2.75 M_{\odot}$ for the companion when $m_{1.4}=1$. This upper limit is indeed higher than the maximum mass expected for a main sequence star belonging to one of the two stellar populations found by Ferraro et al. (2009) in Terzan 5. One has in fact $m_{2} \lesssim 0.95$ if the companion of J17480 belongs to the older population ( $t=10 \mathrm{Gyr}$, D'Antona et al. 2010), while $m_{2} \lesssim 1.2$ and $m_{2} \lesssim 1.5$ if it belongs to a younger population of 6 and 4 Gyr, respectively (D'Antona, priv. comm.). We conclude that a reasonable upper limit for the companion-star mass is $1.5 M_{\odot}$, possibly a main sequence or a slightly evolved star.

Acknowledgements. This work is supported by the Italian Space Agency, ASIINAF I/088/06/0 contract for High Energy Astrophysics, as well as by the operating program of Regione Sardegna (European Social Fund 2007-2013), L.R.7/2007, "Promotion of scientific research and technological innovation in Sardinia". We thank F. D'Antona for providing the mass estimates of main sequence stars in Terzan 5, and the referee for the prompt reply and useful comments.

\section{References}

Altamirano, D., Homan, J., Linares, M., et al. 2010a, ATel, 2952, 1 Altamirano, D., Watts, A., Kalamkar, M., et al. 2010b, ATel, 2932, 1 Bhattacharya, D., \& van den Heuvel, E. P. J. 1991, Phys. Rep., 203, 1 Bordas, P., Kuulkers, E., Alfondo Garzon, J., et al. 2010, ATel, 2919, 1 Bozzo, E., Ferrigno, C., \& Bordas, P. 2010, ATel, 2922, 1

Burderi, L., Possenti, A., D’Antona, F., et al. 2001, ApJ, 560, L71 Burderi, L., Di Salvo, T., Menna, M. T., et al. 2006, ApJ, 653, L133 Burderi, L., Di Salvo, T., Lavagetto, G., et al. 2007, ApJ, 657, 961 Cohn, H. N., Lugger, P. M., Grindlay, J. E., et al. 2002, ApJ, 571, 818 Cui, W. 1997, ApJ, 482, L163

D'Antona, F., Caloi, V., \& Ventura, P. 2010, MNRAS, 405, 2295

Deeter, J. E., Boynton, P. E., \& Pravdo, S. H. 1981, ApJ, 247, 1003 Eggleton, P. P. 1983, ApJ, 268, 368

Ferraro, F. R., Dalessandro, E., Mucciarelli, A., et al. 2009, Nature, 462, 483 Heinke, C. O., Linares, M., \& Gladstone, J. 2010, ATel, 2933, 1 Heinke, C. O., Wijnands, R., Cohn, H. N., et al. 2006, ApJ, 651, 1098 Hessels, J. W. T., Ransom, S. M., Stairs, I. H., et al. 2006, Science, 311, 1901 Jonker, P. G., \& van der Klis, M. 2001, ApJ, 553, L43 Kennea, J. A., Evans, P. A., \& Beardmore, A. P. 2010, ATel, 2937, 1 Leahy, D. A. 1987, A\&A, 180, 275

Makishima, K., Ohashi, T., Inoue, H., et al. 1981, ApJ, 247, L23 Meylan, G., \& Heggie, D. C. 1997, A\&ARv, 8, 1

Ortolani, S., Barbuy, B., Bica, E., et al. 2007, A\&A, 470, 1043 Papitto, A., Burderi, L., D'Aì, A., et al. 2010, ATel, 2939, 1 Pooley, D., Homan, J., Heinke, C., et al. 2010, ATel, 2974, 1 Poutanen, J., \& Svensson, R. 1996, ApJ, 470, 249

Ransom, S. M., Hessels, J. W. T., Stairs, I. H., et al. 2005, Science, 307, 892 Riggio, A., Di Salvo, T., Burderi, L., et al. 2008, ApJ, 678, 1273 Strohmayer, T. E., \& Markwardt, C. B. 2010, ATel, 2929, 1 Strohmayer, T. E., Markwardt, C. B., Pereira, B., et al. 2010, ATel, 2946, 1 Wijnands, R., \& van der Klis, M. 1998, Nature, 394, 344 